\title{
REMARKS ON APPROXIMATION METHODS IN DEGREE THEORY
}

\author{
W. KRYSZEWSKI, B. PRZERADZKI AND S. WEREŃSKI
}

\begin{abstract}
An approximative approach to a generalized theory of the topological mapping degree is presented. Some new wide classes of operators acting in Banach spaces, which include $A$-proper mappings of Petryshyn, are introduced and studied from the viewpoint of the homotopic properties of the topological degree. The results are applied in some existence aspects of abstract nonlinear equations.
\end{abstract}

\section{INTRODUCTION}

One of the main tools for the study of existence problems for a nonlinear equation $f(y)=x$ is the so-called topological degree of a mapping $f$. Generally speaking, the degree of $f$ is an element of a suitably chosen abelian group $G$ which is invariant under homotopies and, in the case when it does not vanish, the equation $f(y)=x$ has at least an approximative solution.

If the map acts in a finite-dimensional space, then assuming the continuity of $f$ we can define the Brouwer degree $[1,14]$, which is integer valued. The direct generalization of the Brouwer degree theory to the infinite-dimensional case needs new bounds on $f$. The first infinite-dimensional theory of degree (due to Leray and Schauder [13]) concerned mappings of the form $f=I-$ $C$ where $I$ is the identity and $C$ is compact. The Leray-Schauder theory is based on the possibility of the uniform approximation of a compact map $C$ by finite-dimensional mapping. Many functional equations (in particular, integral equations and boundary value problems for partial differential equations) may be reduced to the form $y-C y=x$, which enables one to apply the LeraySchauder theory.

For generalizations of these results, one needs some extensions of the class of operators. There are two directions in the attempts to extend the class of Leray-Schauder maps. In the first, a mapping $C$ (not compact) is replaced by a compact operator $\widetilde{C}$ connected with $C$ in such a way that the solvability of $y-\widetilde{C} y=x$ implies the same for $y-C y=x$ (see $[17,21,14])$. In the second, the equation $f(x)=x$ is replaced by a sequence of finite-dimensional equations $f_{n}(y)=x_{n}$ where $\left(x_{n}\right)$ approximates $x$ and almost all Brouwer degrees for $f_{n}$ and $x_{n}$ are defined. The connection between $f$ and $\left(f_{n}\right)$ is

Received by the editors March 2, 1988.

1980 Mathematics Subject Classification (1985 Revision). Primary 47H17, 47H15; Secondary 47H09, 47H10. 
such that the sequence of the Brouwer degrees of $f_{n}$ gives information on the solvability of the equation $f(y)=x$. A fairly general and detailed study of the degree theory via approximation processes was given in Browder's paper [2].

We would like to set in order the generalization containing the theory of $A$ proper mappings of Petryshyn [19, 18] (see also [3], [4] and e.g., [22] for some applications) and the theory of $D C$-mappings introduced by Nowak [16]. This last family of operators was studied by the present authors in [9-12, 20, 23]. The above-mentioned families of maps contain all Leray-Schauder operators, but they do not coincide. We found a sufficiently large class which contains both continuous $A$-proper mappings in spaces with projectional schemes and $D C$ maps, and we have defined a topological degree for it. This degree is consistent with the earlier definitions. In this paper we present the construction of the class and the degree. One can see that successive extensions of the classes of operators complicate the properties of mappings and their degrees and make application more difficult. On the other hand, we show the role of a projectional scheme; projectors enable one to extend the studied class greatly but the degree loses a topological nature.

In this paper, $D C$-mappings are called admissible maps, or $A$-maps.

\section{The ClASSIFICATION OF SEQUENCES AND MAPS}

Let $X$ be a Banach space and let $\left(X_{n}\right)_{n \in N}$ be an increasing sequence of finitedimensional linear subspaces of $X$. The sequence $\left(X_{n}\right)$ is called a filtration in $X$ (see [16]) if

$$
\mathrm{cl} \bigcup_{n \in N} X_{n}=X
$$

Obviously, every space with a filtration is separable and, conversely, every separable Banach space admits a filtration.

Fix a filtration $\left(X_{n}\right)$ in $X$ and a sequence $\left(z_{n}\right)$ of points of $X$. We shall say that a subsequence $\left(z_{n_{k}}\right)$ is associated with a point $z \in X$ when

$$
\left\|z_{n_{k}}-z\right\|-d\left(z_{n_{k}}, X_{n_{k}}\right) \rightarrow 0
$$

where $d(\cdot, \cdot)$ denotes the distance between point and set. If it is so, we write $\left(z_{n_{k}}\right) \vdash z$. Let us note that if $z$ is a cluster point of $\left(z_{n}\right)$, then a certain subsequence $\left(z_{n_{k}}\right)$ is associated with $z$. However, the converse is false. There are subsequences which are associated with points that are not cluster points for the sequence.

Example 1. Let $X$ be a separable Hilbert space and let $\left\{e_{n}: n \in N\right\}$ be its orthonormal basis. The filtration $\left(X_{n}\right)$ is defined by

$$
X_{n}=\operatorname{span}\left\{e_{i}: i \leq n\right\} \text {. }
$$

The sequence $\left(z_{n}\right)$, where $z_{n}=e_{n+1}$, is associated with $z=0$, but it has no cluster points. At the same time, we see that the relation of association strongly 
depends on the numeration of the sequence since $\left(z_{n-1}\right)$ is not associated with 0 .

Obviously, if $z_{n} \in X_{n}$ for large $n$, then each point $z$, having a subsequence $\left(z_{n_{k}}\right)$ such that $\left(z_{n_{k}}\right) \vdash z$, is a cluster point of $\left(z_{n}\right)$.

As has been mentioned, we shall use projectionally complete schemes. Assume that there is a sequence $\left(P_{n}\right)$ of linear projectors $P_{n}: X \rightarrow X_{n}$ in the Banach space $X$ with the filtration $\left(X_{n}\right)$, and

$$
\sup _{n}\left\|P_{n}\right\|<\infty \text {. }
$$

Then the pair $\left(\left(X_{n}\right),\left(P_{n}\right)\right)$ is called a projectionally complete scheme in $X$ (see [18]). Due to the Banach-Steinhaus theorem, condition (3) is equivalent to

$$
P_{n} x \rightarrow x, \quad x \in X .
$$

If the space $X$ has a Schauder basis $\left\{y_{j}: j \in N\right\}$ then the formulae

$$
X_{n}=\operatorname{span}\left\{y_{1}, \ldots, y_{n}\right\}, \quad P_{n}\left(\sum_{j=1}^{\infty} \alpha_{j} y_{j}\right)=\sum_{j=1}^{n} \alpha_{j} y_{j}
$$

determine a projectionally complete scheme. Conversely, if $\left(\left(X_{n}\right),\left(P_{n}\right)\right)$ is a projectionally complete scheme and $P_{n} P_{m}=P_{\min (m, n)}$ for each $m, n$, then there exists a Schauder basis which defines the scheme in the above way.

Now we are in a position to classify sequences in a Banach space $X$ with a filtration $\left(X_{n}\right)$. For the last part of the following definition, it will be necessary to fix a projectionally complete scheme in $X$.

Definition. A sequence $\left(z_{n}\right)$ is said to be

(i) compact (or $C$-sequence),

(ii) admissible (or $A$-sequence),

(iii) filtrated (or $F$-sequence),

(iv) projectionally filtrated (or $P F$-sequence),

if, for each of its subsequences $\left(z_{n_{k}}\right)$,

(i) there exists a convergent subsequence $\left(z_{n_{k(1)}}\right)$,

(ii) there exists a subsequence $\left(z_{n_{k(j)}}\right)$ such that

$$
d\left(z_{n_{k(1)}}, X_{n_{k(1)}}\right) \rightarrow 0
$$

(iii) the condition $\left(z_{n_{k}}\right) \vdash z$ (for some $z$ ) implies that there exists a subsequence $\left(z_{n_{k(1)}}\right)$ converging to $z$,

(iv) the condition $\left(P_{n_{k}} z_{n_{k}}\right) \vdash z$ (for some $z$ ) implies that there exists a subsequence $\left(z_{n_{k(1)}}\right)$ converging to $z$.

Remark. The definitions of $C-, A$-, and $P F$-sequences can be simplified (they are given in such a form only for symmetry). Namely, $\left(z_{n}\right)$ is a $C$-sequence iff the set $\left\{z_{n}: n \in N\right\}$ is relatively compact; $\left(z_{n}\right)$ is an $A$-sequence iff

$$
d\left(z_{n}, X_{n}\right) \rightarrow 0
$$


$\left(z_{n}\right)$ is a $P F$-sequence iff, for each subsequence $\left(z_{n_{k}}\right), P_{n_{k}} z_{n_{k}} \rightarrow z$ implies that $z$ is a cluster point of $\left(z_{n_{k}}\right)$.

It is easy to see that every $C$-sequence is an $A$-sequence, and every $A$ sequence is both an $F$-sequence and $P F$-sequence.

The class of $A$-sequences is essentially larger than the class of $C$-sequences since it contains all sequences $\left(z_{n}\right)$ with the property $z_{n} \in X_{n}, n \in N$. There are examples of $P F$-sequences and $F$-sequences which are not admissible.

Example 2. Let $X=c_{0}$ be the space of sequences converging to 0 , with the filtration $\left(X_{n}\right)$ given by the Schauder basis $\left\{e_{n}: n \in N\right\}$ where $e_{n}=\left(\delta_{k n}\right)_{k \in N}$, and with the natural projectors $\left(P_{n}\right)$. Let $0<r<1$ and $z_{n}=e_{n}+r e_{n+1}$ for $n \in N$. Then

$$
d\left(z_{n}, X_{n}\right)=r
$$

thus $\left(z_{n}\right)$ is not an $A$-sequence. At the same time, if $\left(z_{n_{k}}\right) \vdash z=\sum t_{n} e_{n}$, then $\left|1-t_{n_{k}}\right| \leq\left\|z_{n_{k}}-z\right\| \rightarrow r$, which contradicts $\left|1-t_{n_{k}}\right| \rightarrow 1$. Hence, for any subsequence $\left(z_{n_{k}}\right)$ there is no $z$ such that $\left(z_{n_{k}}\right) \vdash z$. Therefore $\left(z_{n}\right)$ is an $F$-sequence. Similarly, $\left(P_{n} z_{n}\right)=\left(e_{n}\right)$ has no cluster points, so $\left(z_{n}\right)$ is a $P F$-sequence as well.

In the space $X$ with an arbitrary projectional scheme $\left(\left(X_{n}\right),\left(P_{n}\right)\right)$, the classes of $F$-sequences and of $P F$-sequences are not comparable. One can see that, for schemes such that

$$
d\left(z, X_{n}\right)=\left\|z-P_{n} z\right\|
$$

for $z \in X$ and $n \in N$, both classes are equal.

Example 3. Let $X,\left(X_{n}\right)$, and $\left\{e_{n}\right\}$ be as in Example 2. We define projectors $P_{n}: X \rightarrow X_{n}, n \geq 2$, in a different way:

$$
P_{n}\left(\sum_{i=0}^{\infty} t_{i} e_{i}\right)=\sum_{i=0}^{n-1} t_{i} e_{i}+\left(t_{n}+2 t_{n+1}\right) e_{n} .
$$

$\left(P_{n}\right)$ satisfies condition (3). Take two sequences

$$
y_{n}=e_{n}-(1-1 / n) e_{n+1}, \quad z_{n}=e_{n}-\frac{1}{2} e_{n+1},
$$

$n \in N$. As $P_{n} y_{n}=(1 / n-1) e_{n}$ and $P_{n} z_{n}=0$, then $\left(y_{n}\right)$ is projectionally filtrated and $\left(z_{n}\right)$ is not. On the other hand, for any $y=\sum t_{i} e_{i}$ such that $\|y\| \leq 1$, we have

$$
\left\|y_{n}-y\right\|=\max \left(\left|1-t_{n}\right|,\left|t_{n+1}-1+\frac{1}{n}\right|, \sup _{i \neq n+1}\left|t_{i}\right|\right) .
$$

Hence $\left\|y_{n}-y\right\| \rightarrow 1$ and $d\left(y_{n}, X_{n}\right)=1-1 / n \rightarrow 1$. So $\left(y_{n}\right) \vdash y$, but $\left(y_{n}\right)$ has no convergent subsequences. Similar arguments (as in Example 2) show that $\left(z_{n_{k}}\right) \vdash z$ is false for any $\left(z_{n_{k}}\right)$ and $z$. Therefore, $\left(y_{n}\right)$ is not filtrated and $\left(z_{n}\right)$ is.

Now we can classify mappings in the unified way. Let $\alpha$ denote one of the symbols $C, A, F, P F$. Fix Banach spaces $X, Y$ with filtrations $\left(X_{n}\right)$ and 
$\left(Y_{n}\right)$, respectively, and a set $D \subset Y$. In the case $\alpha=P F$, let us also fix projectors $P_{n}, n \in N$, satisfying (3).

Definition. We shall say that $f: D \rightarrow X$ is an $\alpha$-mapping if, for any $y_{n} \in$ $D \cap Y_{n}, n \in N$, the sequence $\left(f\left(y_{n}\right)\right)$ is an $\alpha$-sequence. i.e.,

The above definition is usually used in the case when $D$ is filtrated by $\left(Y_{n}\right)$,

$$
D \subset \operatorname{cl} \bigcup_{n \in N}\left(D \cap Y_{n}\right),
$$

and $f$ is continuous. If $G$ is open in $Y$, then $G, \operatorname{cl} G$, and $\operatorname{fr} G$ are examples of sets satisfying (7). Under the above assumptions, the class of $C$-maps equals the class of compact operators and $A$-mappings are identical with $D C$-maps.

From now on, we assume that all $\alpha$-maps are continuous and their domains satisfy (7).

The following relations are obvious:

$$
\{C \text {-mappings }\} \subset\{A \text {-mappings }\} \subset\left\{\begin{array}{l}
\{F \text {-mappings }\}, \\
\{P F \text {-mappings }\} .
\end{array}\right.
$$

The simplest example of an $A$-map which is not compact is the identity $I$. It can be shown that the family of $A$-maps is closed with respect to addition and multiplication by continuous and bounded functions and that the superposition of $A$-maps belongs to the class provided that the outer factor is uniformly continuous (see [11,12]). Moreover, any $A$-map can be uniformly approximated by operators $f$ with the property $f\left(Y_{n}\right) \subset X_{n}$ for sufficiently large $n \in N$ (see $[11,20])$.

Recall that $f: D \rightarrow X$ is said to be $A$-proper if, for any bounded sequence $\left(y_{n_{k}}\right)$ such that $y_{n_{k}} \in Y_{n_{k}} \cap D$ and $P_{n_{k}} f\left(y_{n_{k}}\right) \rightarrow z$, there exists a subsequence $y_{n_{k(J)}} \rightarrow y$ with $f(y)=z$. It is evident that every $A$-proper map is projectionally filtrated. Since, in a space with a projectionally complete scheme, the classes of $A$-maps and of $A$-proper maps do not coincide, $P F$-mappings are a natural generalization of both these classes.

Example 4. Let $X,\left(X_{n}\right),\left(P_{n}\right)$, and $\left\{e_{n}\right\}$ be as in Example 2. Define $g: X \rightarrow$ $X$ be the formula

$$
g\left(\sum_{i=0}^{\infty} t_{i} e_{i}\right)=r \sum_{i=0}^{\infty} t_{i} e_{i+1}
$$

where $0<r<1$. Then $\|g(x)-g(y)\|=r\|x-y\|$, so $g$ is an $r$-set contraction. Due to the theorem of Petryshyn [19], $f=I-g$ is $A$-proper on any bounded set $D \subset X$. But for $x_{n} \in X_{n}, n \in N$, we have $d\left(f\left(x_{n}\right), X_{n}\right)=r$. Therefore $f$ is not admissible.

Example 5. Let $X$ be a separable Hilbert space, and let $\left(e_{n}\right)_{n=0}^{\infty}$ be an orthonormal basis in $X, X_{n}=\operatorname{span}\left\{e_{1}, \ldots, e_{n}\right\}$. Denote by $P_{n}, n \in N$, the 
orthonormal projection onto $X_{n}$ and put

$$
f\left(\sum_{i=1}^{\infty} t_{i} e_{i}\right)=t_{1} e_{1}+t_{2} e_{2}+\sum_{i=3}^{\infty}\left(t_{i}+t_{i-1}\right) e_{i} .
$$

For $f\left(X_{n}\right) \subset X_{n}, n \in N, f: X \rightarrow X$ is an $A$-mapping.

On the other hand, the sequence

$$
x^{(n)}=n^{-1 / 2}\left(e_{1}-e_{2}+\cdots+(-1)^{n} e_{n}\right) \in X_{n} \backslash X_{n-1}
$$

satisfies $\left\|x^{(n)}\right\|=1$ and

$$
P_{n} f\left(x^{(n)}\right)=n^{-1 / 2}\left(e_{1}-e_{2}+\cdots+(-1)^{n+1} e_{n}\right) \rightarrow 0 .
$$

If $f$ were $A$-proper, the sequence $\left(x^{(n)}\right)$ would contain a convergent subsequence. But, for $n>m$,

$$
\left\|x^{(n)}-x^{(m)}\right\|=\sum_{i=1}^{m}\left(n^{-1 / 2}-m^{-1 / 2}\right)+\sum_{i=m+1}^{n} n^{-1} \geq \frac{n-m}{n},
$$

so there is no convergent subsequence and $f$ is not $A$-proper.

The following theorem fixes a place for $A$-proper maps in our classification.

Theorem 1. Let $D$ be a bounded set in $Y$. A projectionally filtrated mapping $f: D \rightarrow X$ is A-proper iff it is proper.

Proof (cf. [10]). It is known from [18] that, under our assumptions, $A$-proper maps are proper.

Suppose that $f$ is a proper $P F$-mapping. Let $y_{n_{k}} \in Y_{n_{k}} \cap D$, where $\left(n_{k}\right)$ is a subsequence of $N$, be such that $P_{n_{k}} f\left(y_{n_{k}}\right) \rightarrow z$. Then $\left(P_{n_{k}} f\left(y_{n_{k}}\right)\right) \vdash z$, so there exists a subsequence $\left(y_{n_{k(j)}}\right)$ such that $f\left(y_{n_{k(j)}}\right) \rightarrow z$. The set $K=$ $\left\{f\left(y_{n_{k(j)}}\right): j \in N\right\} \cup\{z\}$ is compact; thus $\left(y_{n_{k(j)}}\right)$ being a subsequence of the compact set $f^{-1}(K)$ has a subsequence, denoted also by $\left(y_{n_{k(j)}}\right)$ for simplicity, converging to some $y \in f^{-1}(K)$. The continuity of $f$ implies $f(y)=z$ and, therefore, $f$ is $A$-proper. Q.E.D.

In the case of the lack of projectors, we have no equivalent of $A$-proper maps. However, filtrated mappings are an essential extensions of the family of $A$-maps.

Example 6. Let $X,\left(X_{n}\right)$, and $\left(e_{n}\right)$ be as in Example 2, and let $D$ be the unit ball in $X$. Define $f: D \rightarrow X$ by

$$
f\left(\sum_{i=0}^{\infty} t_{i} e_{i}\right)=\left|t_{0}\right| e_{0}+\sum_{i=1}^{\infty}\left(r\left|t_{i-1}\right|+\left|t_{i}\right|\right) e_{i}
$$

where $0<r<1$. Obviously, $f\left(e_{n}\right)=e_{n}+r e_{n+1}$, so $f$ is not admissible. Meanwhile, for any sequence

$$
\left(x^{(n)}\right) \subset D, \quad x^{(n)}=\sum_{i=0}^{n} t_{i}^{(n)} e_{i} \in X_{n}
$$


we have

$$
d\left(f\left(x^{(n)}\right), X_{n}\right)=r\left|t_{n}^{(n)}\right| .
$$

If $t_{n_{k}}^{\left(n_{k}\right)} \rightarrow 0$ for a subsequence $\left(n_{k}\right)$, then the condition $\left(f\left(x^{\left(n_{k}\right)}\right)\right) \vdash z$ is equivalent to $f\left(x^{\left(n_{k}\right)}\right) \rightarrow z$.

Instead, there is an $\varepsilon>0$ such that $\left|t_{n}^{(n)}\right|>\varepsilon$, then the condition

$$
\left(f\left(x^{\left(n_{k}\right)}\right)\right) \vdash z=\sum_{i=0}^{\infty} s_{i} e_{i}
$$

implies

$$
|r| t_{n_{k}-1}^{\left(n_{k}\right)}|+| t_{n_{k}}^{\left(n_{k}\right)}\left|-s_{n_{k}}\right|-r\left|t_{n_{k}}^{\left(n_{k}\right)}\right| \rightarrow 0
$$

which is impossible since almost all terms of this sequence are greater than $\frac{1}{2}(1-r) \varepsilon>0$. Therefore $f$ is a filtrated map.

The above examples of mappings are rather artificial and were constructed only for visualizing the definitions. The class of $P F$-maps includes all $A$-proper mappings and this gives a long list of natural examples (see [18]). Admissible mappings are both $F$ - and $P F$-maps, and this enables us to give at least two examples (cf. [10-12]).

Example 7. Let $Y=H^{k}(\Omega)$ be the Sobolev space of functions defined on an open and bounded set $\Omega \subset R^{1}$, with square integrable derivatives (in the distribution sense) up to the order $k$. Let $Y_{n}, n \in N$, be a subspace of $Y$ consisting of polynomials of degree $\leq n$. Similarly, $X=H^{0}(\Omega), X_{n}=Y_{n}$ for $n \in N$. Fix polynomials $Q_{\alpha}$ of $l$ variables, where $\alpha=\left(\alpha_{1}, \ldots, \alpha_{l}\right) \in N^{l}$ and $|\alpha|=\sum \alpha_{i} \leq k$, and a continuous function $K: \operatorname{cl} \Omega \times \operatorname{cl} \Omega \times R \rightarrow R$. Then the operator $f$ given by

$$
f(x)(t)=\sum_{\alpha} Q_{\alpha}(t) D^{\alpha} x(t)+\int_{\Omega} K(t, s, x(s)) d s
$$

is an $A$-map on any bounded set $D \subset Y$ with values in $X$, since the first summand transform $Y_{n}$ into $X_{n}$ and the second one is compact.

Example 8. Let $X$ be the space consisting of all continuous functions $x$ : $\operatorname{cl} \Omega$ $\rightarrow R$ vanishing at infinity, where $\Omega$ is open (not necessarily bounded) in $R^{l}$. Let $K:\langle 0, \infty) \rightarrow R$ be a Lipschitz function monotonic for large arguments. Moreover, we claim that

$$
\int_{0}^{\infty}|K(\lambda)| \lambda^{l} d \lambda<\infty
$$

Let $L: \operatorname{cl} \Omega \times R \rightarrow R$ be a measurable function satisfying the Lipschitz condition with respect to the second variable and such that there exists a continuous function $\varphi: R \rightarrow R$ with

$$
\begin{gathered}
\varphi(0)=0, \\
|L(s, \lambda)|=\varphi(\lambda), \quad s \in \operatorname{cl} \Omega, \lambda \in R .
\end{gathered}
$$


Then the Hammerstein operator

$$
f(x)(t)=\int_{\Omega} K(\|t-s\|) L(s, x(s)) d s
$$

transforms $X$ into $X$ continuously. In general, $f$ (restricted to a bounded set $D \subset X)$ need not be compact:

$$
\Omega=(0, \infty) \subset R^{1}, \quad K(\lambda)=\exp (-\lambda), \quad L(s, \lambda)=\lambda .
$$

However, one can construct filtrations $\left(Y_{n}\right)$ and $\left(X_{n}\right)$, both in $X$, such that $f: D \rightarrow X$ is admissible with respect to the filtration $\left(Y_{n}\right)$ in the domain and $\left(X_{n}\right)$ in the range, satisfying the condition $\operatorname{dim} Y_{n}=\operatorname{dim} X_{n}, n \in N$, essential for the degree theory constructed below.

To that end, the set $\operatorname{cl} \Omega$ is represented as a union of an increasing sequence of bounded sets $\left(\mathrm{cl} \Omega_{n}\right)$, the space $X$ is filtrated by the sequences $Z_{n}$ consisting of functions vanishing outside $\Omega_{n}$. The operator $f$ is compact on $D \cap Z_{n}$ for any $n \in N$. The filtration $\left(X_{n}\right)$ is constructed on the basis of a finite $n^{-1}$-net of $f\left(D \cap Z_{n}\right)$. Next we take an arbitrary filtration $\left(Y_{n}\right)$ in $X$ such that $Y_{n}$ is a subspace of $Z_{n}$ and $\operatorname{dim} Y_{n}=\operatorname{dim} X_{n}$ (see also [12], and [10] for a certain generalization).

\section{APPROXIMATIONS OF MAPPINGS}

The essence of the approximation method of solving $f(y)=x$ lies in the construction of auxiliary finite-dimensional equations $f_{n}\left(y_{n}\right)=x_{n}$ which can be solved and, next, the construction of a solution to the initial equation with the help of the auxiliary solutions.

If $Y, X$ are Banach spaces with filtrations $\left(Y_{n}\right),\left(X_{n}\right)$, respectively, and if a sequence $\left(P_{n}: X \rightarrow X_{n}\right)$ of linear projectors satisfying (3) is given, then the natural choice of $\left(f_{n}\right)$ is $f_{n}=P_{n} f \mid Y_{n}$ for $n \in N$. Now, it is also clear that the assumption $\operatorname{dim} Y_{n}=\operatorname{dim} X_{n}$ seems to be necessary.

The following theorem explains which sequences $\left(f_{n}\right)$ "approximate" $f$ when there are no projectors.

Theorem 2. Let $f: D \rightarrow X$ be continuous. Then there exists a sequence $\left(f_{n}\right)$ of continuous maps $f_{n}: D \cap Y_{n} \rightarrow X_{n}, n \in N$, such that

$$
\lim _{n \rightarrow \infty} \sup _{y_{n} \in D \cap Y_{n}}\left[\left\|f\left(y_{n}\right)-f_{n}\left(y_{n}\right)\right\|-d\left(f\left(y_{n}\right), X_{n}\right)\right]=0 .
$$

Proof. Fix a sequence of positive numbers $\left(\varepsilon_{n}\right)$ converging to 0 and let $n \in N$. For any $u_{n} \in D \cap Y_{n}$, put

$$
U\left(u_{n}\right)=X_{n} \cap B\left(f\left(u_{n}\right), d\left(f\left(u_{n}\right), X_{n}\right)+\varepsilon_{n} / 2\right)
$$

(where $B(z, r)$ is the open ball with center $z$ and radius $r$ ). The set $U\left(u_{n}\right)$ is open in $X_{n}$ and nonempty. Let $z_{n}\left(u_{n}\right) \in U\left(u_{n}\right)$. Take a locally finite partition of unity $\left\{\varphi_{u_{n}}: u_{n} \in D \cap Y_{n}\right\}$ subordinate to the open cover

$$
\left\{f^{-1}\left(B\left(f\left(u_{n}\right), \varepsilon_{n} / 2\right)\right): u_{n} \in D \cap Y_{n}\right\}
$$


of $D \cap Y_{n}$. The mapping $f_{n}: D \cap Y_{n} \rightarrow X_{n}$ is defined by the formula

$$
f_{n}\left(y_{n}\right)=\sum_{u_{n}} \varphi_{u_{n}}\left(y_{n}\right) z_{n}\left(u_{n}\right)
$$

For any $y_{n} \in D \cap Y_{n}$, we have

$$
\left\|f\left(y_{n}\right)-f_{n}\left(y_{n}\right)\right\| \leq \sum_{u_{n}} \varphi_{u_{n}}\left(y_{n}\right)\left\|f\left(y_{n}\right)-z_{n}\left(u_{n}\right)\right\| .
$$

If $\varphi_{u_{n}}\left(y_{n}\right) \neq 0$, then $f\left(y_{n}\right) \in B\left(f\left(u_{n}\right), \varepsilon_{n} / 2\right)$; so

$$
\begin{aligned}
\left\|f\left(y_{n}\right)-z_{n}\left(u_{n}\right)\right\| & \leq\left\|f\left(y_{n}\right)-f\left(u_{n}\right)\right\|+\left\|f\left(u_{n}\right)-z_{n}\left(u_{n}\right)\right\| \\
& \leq d\left(f\left(u_{n}\right), X_{n}\right)+\varepsilon_{n} .
\end{aligned}
$$

Hence

$$
0 \leq\left\|f\left(y_{n}\right)-f_{n}\left(y_{n}\right)\right\|-d\left(f\left(y_{n}\right), X_{n}\right) \leq \varepsilon_{n},
$$

which ends the proof. Q.E.D.

Each sequence $\left(f_{n}\right)$ satisfying (8) is said to be associated with the map $f$. Theorem 2 can be strengthened. There is a map $f_{n}$ such that

$$
\left\|f\left(y_{n}\right)-f_{n}\left(y_{n}\right)\right\|=d\left(f\left(y_{n}\right), X_{n}\right)
$$

for $n \in N$ and $y_{n} \in D \cap Y_{n}$. This can be shown by means of the Michael selection theorem; it is less elementary. Notice also that condition (9) uniquely determines the sequence $\left(f_{n}\right)$ in a uniformly convex space $X$. In the sequel, only condition (8) will be needed.

Unfortunately, the procedure described at the beginning of this section does not work for every map $f$ and a sequence $\left(f_{n}\right)$ associated with it. It does work if $f$ belongs to one of the classes $\alpha(\alpha=C, A, F, P F)$. Namely, we have the following

Theorem 3. (i) Under the assumptions of Theorem 2, if $f: D \rightarrow X$ is an $F$ mapping, then, any sequence $\left(f_{n}\right)$ associated with $f$, the conditions

$$
\begin{gathered}
x \in \operatorname{cl} f(D), \\
x=\lim x_{n_{k}} \quad \text { where } x_{n_{k}} \in f_{n_{k}}\left(D \cap Y_{n_{k}}\right)
\end{gathered}
$$

are equivalent.

(ii) If $\left(P_{n}\right)$ is a sequence of linear projectors satisfying (3) and $f$ is a PF-map, then condition (a) is equivalent to

$$
x=\lim x_{n_{k}} \text { where } x_{n_{k}} \in P_{n_{k}} f\left(D \cap Y_{n_{k}}\right) .
$$

Proof. (i) Suppose $f: D \rightarrow X$ is filtrated and $\left(f_{n}\right)$ is a sequence associated with $f$. If $x \in \operatorname{cl} f(D)$, then, by (7), there is a sequence $y_{n} \in D \cap Y_{n}, n \in$ $N$, such that $x=\lim f\left(y_{n}\right)$. Due to (8) and $d\left(f\left(y_{n}\right), X_{n}\right) \rightarrow 0$, we obtain $\left\|f\left(y_{n}\right)-f_{n}\left(y_{n}\right)\right\| \rightarrow 0$. Hence $x=\lim f_{n}\left(y_{n}\right)$.

Conversely, if $x=\lim f_{n_{k}}\left(y_{n_{k}}\right)$ where $y_{n_{k}} \in D \cap Y_{n_{k}}$, then by (8)

$$
\left\|f\left(y_{n_{k}}\right)-x\right\|-d\left(f\left(y_{n_{k}}\right), X_{n_{k}}\right) \rightarrow 0 \text {. }
$$


This means that $\left(f\left(y_{n_{k}}\right) \vdash x\right.$. By the definition, there is a subsequence $\left(y_{n_{k(j)}}\right)$ such that $f\left(y_{n_{k(j)}}\right) \rightarrow x$, so $x \in \operatorname{cl} f(D)$.

(ii) If $x \in \operatorname{cl} f(D)$, then $x=\lim f\left(y_{n}\right)$ where $y_{n} \in D \cap Y_{n}$. By (3) and the inequality

$$
\left\|f\left(y_{n}\right)-P_{n} f\left(y_{n}\right)\right\| \leq\left(\left\|P_{n}\right\|+1\right) d\left(f\left(y_{n}\right), X_{n}\right),
$$

we have $P_{n} f\left(y_{n}\right) \rightarrow x$. The converse is obvious. Q.E.D.

The above theorem enables us to obtain an approximate solution to the equation $f(y)=x$ if the solution of the auxiliary equations $f_{n_{k}}\left(y_{n_{k}}\right)=x_{n_{k}}$ $\left(x_{n_{k}} \rightarrow x\right)$ is given. If it is known from anywhere else that the set $f(D)$ is closed, then there exists an exact solution. When, for instance, $f$ is proper and $D$ is closed, this solution is also the limit of the sequence of the auxiliary solutions. Such a situation corresponds to the theory of $A$-proper mappings of Petryshyn.

In the sequel, if a mapping $f$ belongs to the class of $F$-maps, we denote by $\left(f_{n}\right)$ an arbitrary sequence associated with $f$, and if $f$ is projectionally filtrated, then $f_{n}=P_{n} f \mid Y_{n}$.

\section{THE DEGREE OF A MAPPING}

Let $Y$ and $X$ be Banach spaces with filtrations $\left(Y_{n}\right)$ and $\left(X_{n}\right)$, respectively, such that

$$
\operatorname{dim} Y_{n}=\operatorname{dim} X_{n}
$$

for $n \geq n_{0}$. We fix orientations in $Y_{n}$ and $X_{n}, n \geq n_{0}$.

Suppose that $D \subset Y$ is open and bounded and take a continuous mapping $f: \operatorname{cl} D \rightarrow X$ and an $x \in X \operatorname{cl} f(\operatorname{fr} D)$. Let $\varepsilon=d(x, f(\operatorname{fr} D))>0$. By (1), there are $n_{1} \geq n_{0}$ and $\bar{x} \in X_{n_{1}}$ such that $\|x-\bar{x}\|<\varepsilon / 2$. Hence $\bar{x} \notin \operatorname{cl} f(\operatorname{fr} D)$.

Assume that $f$ is an $F$-map (resp. $P F$-mapping). We claim that there exists $n_{2} \geq n_{1}$ such that

$$
\bar{x} \notin f_{n}\left(\operatorname{fr}\left(D \cap Y_{n}\right)\right)
$$

for any $n \geq n_{2}$, where $\left(f_{n}\right)$ is an arbitrary sequence associated with $f$ (resp. $\left.f_{n}=P_{n} f \mid D \cap Y_{n}\right)$. Suppose to the contrary that there exists a sequence $\left(y_{n_{k}}\right)$, $y_{n_{k}} \in \operatorname{fr}\left(D \cap Y_{n_{k}}\right) \subset \operatorname{fr} D \cap Y_{n_{k}}$, such that $f_{n_{k}}\left(y_{n_{k}}\right)=\bar{x}$. Then, by Theorem 3, $\bar{x} \in \operatorname{cl} f(\operatorname{fr} D)$.

Therefore, we can define

$$
s_{n}= \begin{cases}\operatorname{deg}\left(f_{n}, D \cap Y_{n}, X\right) & \text { for } n \geq n_{2}, \\ 0 & \text { for } n<n_{2},\end{cases}
$$

where deg stands for the finite-dimensional Brouwer degree [1, 14]. Let us denote

$$
\mathscr{G}=\prod_{n=1}^{\infty} Z / \bigoplus_{n=1}^{\infty} Z
$$


(i.e., $\mathscr{G}$ is a factor group of the group of integer-valued infinite sequences and its subgroup of finite sequences) and $\nu: \prod_{i=1}^{\infty} Z \rightarrow \mathscr{G}$-the canonical homomorphism.

An element

$$
\operatorname{Deg}(f, D, x)=\nu\left(\left(s_{n}\right)_{n=1}^{\infty}\right)
$$

of the group $\mathscr{G}$ is called the degree of the F-mapping (resp. $P F$-mapping) $f$ on $D$ at the point $x$.

We shall show that the definition is correct; i.e., it does not depend on the choice of $\bar{x}$ and the sequence $\left(f_{n}\right)$ (in the case of a $P F$-map, one needs only the independence of the choice of $\bar{x}$ ).

Let $\bar{x}^{\prime} \in X_{n_{1}^{\prime}}$ be such that $\left\|\bar{x}^{\prime}-x\right\|<\varepsilon / 2$, and let $\left(f_{n}^{\prime}\right)$ be another sequence associated with $f$. Suppose that the numbers $s_{n}$ and $s_{n}^{\prime}$ are defined for $n \geq N_{0}$ as above $\left(s_{n}^{\prime}\right.$ for $f_{n}^{\prime}$ and $\left.\bar{x}^{\prime}\right)$. Let $h_{n}:\left(\operatorname{cl} D \cap Y_{n}\right) \times\langle 0,1\rangle \rightarrow X_{n}, n \geq N_{0}$, be a linear homotopy

$$
h_{n}\left(y_{n}, t\right)=(1-t) f_{n}\left(y_{n}\right)+t f_{n}^{\prime}\left(y_{n}\right),
$$

and let $x:\langle 0,1\rangle \rightarrow X_{N_{0}}$ be a map given by the formula

$$
x(t)=(1-t) \bar{x}+t \bar{x}^{\prime} .
$$

We shall show that, for sufficiently large $n$ and any $t \in\langle 0,1\rangle$,

$$
x(t) \notin\left\{h_{n}\left(y_{n}, t\right): y_{n} \in \operatorname{fr} D \cap Y_{n}\right\} .
$$

Suppose it is not so. Then there exist sequences $\left(y_{n_{k}}\right)$ and $\left(t_{n_{k}}\right) \subset\langle 0,1\rangle$ such that $y_{n_{k}} \in \operatorname{fr} D \cap Y_{n_{k}}$ and

$$
h_{n_{k}}\left(y_{n_{k}}, t_{n_{k}}\right)=x\left(t_{n_{k}}\right) .
$$

For $n_{k} \geq N_{0}$, we have

$$
\begin{aligned}
0 \leq & \left\|x\left(t_{n_{k}}\right)-f\left(y_{n_{k}}\right)\right\|-d\left(f\left(y_{n_{k}}\right), X_{n_{k}}\right) \\
= & \left\|\left(1-t_{n_{k}}\right)\left[f_{n_{k}}\left(y_{n_{k}}\right)-f\left(y_{n_{k}}\right)\right]+t_{n_{k}}\left[f_{n_{k}}^{\prime}\left(y_{n_{k}}\right)-f\left(y_{n_{k}}\right)\right]\right\|-d\left(f\left(y_{n_{k}}\right), X_{n_{k}}\right) \\
\leq & \left(1-t_{n_{k}}\right)\left[\left\|f_{n_{k}}\left(y_{n_{k}}\right)-f\left(y_{n_{k}}\right)\right\|-d\left(f\left(y_{n_{k}}\right), X_{n_{k}}\right)\right] \\
& +t_{n_{k}}\left[\left\|f_{n_{k}}^{\prime}\left(y_{n_{k}}\right)-f\left(y_{n_{k}}\right)\right\|-d\left(f\left(y_{n_{k}}\right), X_{n_{k}}\right)\right] \rightarrow 0
\end{aligned}
$$

as $k \rightarrow \infty$. Without loss of generality one may assume that $t_{n_{k}} \rightarrow t_{0} \in\langle 0,1\rangle$. Therefore $\left(f\left(y_{n_{k}}\right)\right) \vdash x\left(t_{0}\right)$. By the definition of an $F$-map, $x\left(t_{0}\right) \in \operatorname{cl} f(\operatorname{fr} D)$, which contradicts $\left\|x\left(t_{0}\right)-x\right\|<\varepsilon / 2$. By the homotopy invariance of the Brouwer degree, we obtain the assertion of the theorem.

The proof for a $P F$-mapping is similar but easier.

The degree introduced above has almost all standard properties. The most important property of any topological degree is homotopy invariance. 
Theorem 4. Let one of the following conditions be satisfied:

(i) $h$ : cl $D \times\langle 0,1\rangle \rightarrow X$ is filtrated (resp. projectionally filtrated) with respect to the filtration $\left(Y_{n} \times \mathbf{R}\right)$ in $Y \times \mathbf{R}$,

(ii) $h: \operatorname{cl} D \times\langle 0,1\rangle \rightarrow X$ is continuous and the map $t \rightarrow h(\cdot, t)$ is continuous as a mapping of $\langle 0,1\rangle$ into the space of $F$-maps (resp. PF-maps) endowed with the uniform convergence topology.

If $x \notin \operatorname{cl} h(\operatorname{fr} D \times\langle 0,1\rangle)$, then the function

$$
t \rightarrow \operatorname{Deg}(h(\cdot, t), D, x)
$$

is constant on $\langle 0,1\rangle$.

Proof. (i) Choose a sequence $h_{n}:\left(\operatorname{cl} D \cap Y_{n}\right) \times\langle 0,1\rangle \rightarrow Y_{n}, n \in N$, associated with $h$. Without loss of generality one may assume that $x \in X_{n_{0}}$. Then there exists $n_{1} \geq n_{0}$ such that

$$
x \notin h_{n}\left(\left(\operatorname{fr} D \cap Y_{n}\right) \times\langle 0,1\rangle\right)
$$

for $n \geq n_{1}$. In fact, the opposite assertion leads to the existence of a sequence $\left(y_{n_{k}}, t_{n_{k}}\right), y_{n_{k}} \in \operatorname{fr} D \cap Y_{n_{k}}, t_{n_{k}} \in\langle 0,1\rangle$, such that

$$
h_{n_{k}}\left(y_{n_{k}}, t_{n_{k}}\right)=x \text {. }
$$

Hence $\left(h\left(y_{n_{k}}, t_{n_{k}}\right)\right) \vdash x$ and, applying Theorem 3, we get a contradiction with the assumption on $x$. Therefore, (14) holds.

But the sequence $\left(h_{n}(\cdot, t)\right)$ is associated with $h(\cdot, t)$ for each $t \in\langle 0,1\rangle$. The homotopy invariance of the Brouwer degree ends the proof.

(ii) Take, as above, a sequence $\left(h_{n}\right)$ associated with $h$. Similarly, we can assume $x \in X_{n_{0}}$. We shall verify (14).

Suppose that there exist $y_{n_{k}} \in \operatorname{fr} D \cap Y_{n_{k}}$ and $t_{n_{k}} \in\langle 0,1\rangle$ for $k=1,2, \ldots$ such that $h_{n_{k}}\left(y_{n_{k}}, t_{n_{k}}\right)=x$. Passing to a subsequence, if it is necessary, one can assume that $t_{n_{k}} \rightarrow t_{0}$. Then

$$
\begin{aligned}
0 \leq & \left\|h\left(y_{n_{k}}, t_{0}\right)-x\right\|-d\left(h\left(y_{n_{k}}, t_{0}\right), X_{n_{k}}\right) \\
\leq & \left\|h\left(y_{n_{k}}, t_{0}\right)-h\left(y_{n_{k}}, t_{n_{k}}\right)\right\|+\left\|h\left(y_{n_{k}}\right)-h_{n_{k}}\left(y_{n_{k}}, t_{n_{k}}\right)\right\| \\
& \quad-d\left(h\left(y_{n_{k}}, t_{n_{k}}\right), X_{n_{k}}\right)+\left\|h\left(y_{n_{k}}, t_{n_{k}}\right)-h_{n_{k}}\left(y_{n_{k}}, t_{0}\right)\right\| \rightarrow 0
\end{aligned}
$$

as $k \rightarrow \infty$ since $\left(h_{n}\right)$ is associated with $h$ and the mapping $t \rightarrow h\left(\cdot, t_{0}\right)$ is continuous. Hence $\left(h\left(y_{n_{k}}, t_{0}\right)\right) \vdash x$ and, since $h\left(\cdot, t_{0}\right)$ is filtrated, $x$ is a cluster point of this sequence, which is impossible.

The assertion of the theorem is a consequence of the homotopy invariance of the Brouwer degree.

The proof for $P F$-maps is similar but simpler. Q.E.D.

Now we shall present the remaining standard properties of the degree.

Theorem 5. Suppose $f: \mathrm{cl} D \rightarrow X$ is an F-mapping (resp. PF-mapping).

(i) If $x$ and $x^{\prime}$ belong to the same component of the set $X \backslash \mathrm{cl} f(\operatorname{fr} D)$, then

$$
\operatorname{Deg}(f, D, x)=\operatorname{Deg}\left(f, D, x^{\prime}\right) \text {. }
$$


(ii) If the degree $\operatorname{Deg}(f, D, x)$ is defined and $\neq 0 \in \mathscr{G}$, then $x \subset \mathrm{cl} f(D)$.

(iii) Let $\left\{D^{(i)}: i=-1,2, \ldots, m\right\}$ be a family of open disjoint subsets of $D$. If

$$
x \notin \operatorname{cl} f\left(\operatorname{cl} D \backslash \bigcup_{i=1}^{m} D^{(i)}\right)
$$

then

$$
\operatorname{Deg}(f, D, x)=\sum_{i=1}^{m} \operatorname{Deg}\left(f, D^{(i)}, x\right) \text {. }
$$

(iv) Let $K$ be a closed subset of $Y$ and $x \in \operatorname{cl} f(K \cup \operatorname{fr} D)$. Then

$$
\operatorname{Deg}(f, D \backslash K, x)=\operatorname{Deg}(f, D, x) .
$$

Proof. (i) is a consequence of Theorem 4(ii) and the fact that $y \rightarrow f(y)-x$ is an $F$-mapping (resp. a $P F$-mapping).

(ii) For any $\varepsilon>0$, there exists $x_{\varepsilon} \in \bigcup_{n=1}^{\infty} X_{n}$ belonging to the same component of $X \backslash \operatorname{cl} f(\operatorname{fr} D)$ as $x$, and such that $\left\|x_{\varepsilon}-x\right\|<\varepsilon$. By this and (i), $\operatorname{Deg}\left(f, D, x_{\varepsilon}\right) \neq 0$. Hence, for any associated sequence $\left(f_{n}\right)$, there are $y_{n_{k}} \in D \cap Y_{n_{k}}, k=1,2, \ldots$, such that $f_{n_{k}}\left(y_{n_{k}}\right)=x_{\varepsilon}$. Due to Theorem 3 and the free choice of $\varepsilon, x \in \mathrm{cl} f(D)$.

(iii) One can assume that $x \in X_{n_{0}}$. If $\left(f_{n}\right)$ is an arbitrary sequence associated with $f$, then

$$
x \notin f_{n}\left(\left(\operatorname{cl} D \backslash \bigcup_{n=1}^{m} D^{(i)}\right) \cap Y_{n}\right)
$$

for sufficiently large $n$. Since

$$
\operatorname{cl}\left(D \cap Y_{n}\right) \backslash \bigcup_{i=1}^{m}\left(D^{(i)} \cap Y_{n}\right) \subset\left(\operatorname{cl} D \backslash \bigcup_{i=1}^{m} D^{(i)}\right) \cap Y_{n},
$$

we can apply the additivity property of the Brouwer degree (see [13]):

$$
\operatorname{deg}\left(f_{n}, D \cap Y_{n}, x\right)=\sum_{i=1}^{m} \operatorname{deg}\left(f_{n}, D^{(i)} \cap Y_{n}, x\right) .
$$

(iv) is an immediate consequence of (iii).

The proof for the $P F$-map runs similarly. Q.E.D.

\section{UNIFORM LIMITS OF $F$ - AND $P F$-MAPS}

Now we shall study a slightly larger class of operators and construct a degree for it. The generalization is similar to that in [7]. ${ }^{1}$ The assumptions on spaces, filtrations, projectors, and domain $D$ are not changed.

Let $g: \operatorname{cl} D \rightarrow X$ be bounded on fr $D$; that is, $\sup _{v \in \text { fr } D}\|g(y)\|=M<\infty$. Take a map $f: \operatorname{cl} D \rightarrow X$ such that $f_{\lambda}=f+\lambda g$ are $F$-mappings (resp. $P F$ mappings) for any $\lambda \in(0, \beta\rangle$ ( $\beta>0$ fixed); in particular $f_{\lambda}$ are continuous. In this case $f$ does not have to be an $F$-mapping (resp. a $P F$-mapping).

${ }^{1}$ It should be noted that Browder, independently of Fitzpatrick, also defined the topological degree for limits of $A$-proper maps. 
Example 9. Let $X$ and $\left(X_{n}\right)$ be as in Example 2. Define

$$
g\left(t_{1}, t_{2}, \ldots\right)=\left(1, t_{1}, t_{2}, \ldots\right)
$$

for $\left(t_{k}\right) \in X=c_{0}$, and $f=I-g$. Then $\left(f\left(e_{n}\right)\right) \vdash 0$, but $\left(e_{n}\right)$ has no cluster points, so $f$ is not filtrated. Similarly, one can show that $f$ is not projectionally filtrated.

On the other hand, $f+\lambda g=I-(1-\lambda) g, \lambda \in(0,1\rangle$, is even an $A$-proper map, being a $(1-\lambda)$-set contraction.

We shall define a degree $\operatorname{Deg}_{g}(f, D, x)$ provided that $x \in X \backslash \operatorname{cl} f(\operatorname{fr} D)$. For the purpose, we prove

Lemma 6. Under the above assumptions, there exists $\gamma, 0<\gamma<\beta$, such that

$$
\operatorname{Deg}\left(f_{\lambda}, D, x\right)=\operatorname{Deg}\left(f_{\lambda^{\prime}}, D, x\right)
$$

for $\lambda, \lambda^{\prime} \in(0, \gamma)$.

Proof. Let $\varepsilon=d(x, f(\operatorname{fr} D))$. Taking $\gamma=\min (\varepsilon / 2 M, \beta)$, we obtain $x \notin$ $\operatorname{cl} f_{\lambda}(\mathrm{fr} D)$ for $\lambda \in(0, \gamma)$. It is easy to see that the homotopy $h(\cdot, t)=f_{t \lambda+(1-t) \lambda^{\prime}}$ satisfies the assumptions of Theorem 4(ii), which ends the proof. Q.E.D.

The natural definition of the degree of $f$

$$
\operatorname{Deg}_{g}(f, D, x)=\lim _{\lambda \rightarrow 0^{+}} \operatorname{Deg}\left(f_{\lambda}, D, x\right)
$$

is correct by the above lemma. Using homotopy arguments, one can see that

$$
\operatorname{Deg}_{g}(f, D, x)=\operatorname{Deg}(f, D, x)
$$

if not only $f+\lambda g, \lambda \in(0, \beta\rangle$, is filtrated (resp. projectionally filtrated) but also $f$ is.

Recall the definition of Petryshyn [18]: $f: \operatorname{cl} D \rightarrow X$ is called a $P_{\gamma}$-compact map if, for any $\lambda>\gamma$, the mapping $f_{\lambda}=f-\lambda I$ is $A$-proper. It is clear that the class of $P_{0}$-compact maps is contained in ours.

The degree $\operatorname{Deg}_{g}$ has all standard properties. Let us only mention the homotopy invariance.

Theorem 7. Let $h:\langle 0,1\rangle \ni t \mapsto h_{t}$ and $g: t \mapsto g_{t}$ be continuous maps of $\langle 0,1\rangle$ into the space of continuous transformations of $\mathrm{cl} D$ into $X$, with the uniform convergence topology, and let

$$
\sup _{t} \sup _{y \in \mathrm{fr} D}\left\|g_{t}(y)\right\|=m<\infty .
$$

If $x \notin \mathrm{cl} \bigcup_{t} h_{t}(\mathrm{fr} D)$ and $h_{t}+\lambda g_{t}$ are $F$-maps (resp. PF-maps) for $t \in\langle 0,1\rangle$ and $\lambda \in(0, \beta\rangle$, then

$$
t \rightarrow \operatorname{Deg}_{g_{t}}\left(h_{t}, D, x\right)
$$

is constant on $\langle 0,1\rangle$. 
The proof is obtained by repeating the arguments of the proof of the above lemma for the mapping $H_{\lambda}(\cdot, t)=f_{t}+\lambda g_{t}$.

\section{FIXED POINT THEOREMS}

In this section we shall give a few results concerning the existence of approximate solutions to the equation $f(x)=x$. We use methods of degree theory. All the following results can also be expressed without changes for $P F$-maps.

Theorem 8. Let $X$ be a Banach space with filtration $\left(X_{n}\right)$, and let $D$ be an open bounded subset of $X$. Suppose that $f: \operatorname{cl} D \rightarrow X$ is continuous and bounded on fr $D$ and that, for any $\lambda \in\left\langle 0, p^{-1}\right\rangle(p>0), I-\lambda f$ is an F-mapping.

If the condition

$$
\inf _{x \in \mathrm{fr} D}\left\|f(x)-p x_{0}-q\left(x-x_{0}\right)\right\|=0, \quad q \leq p,
$$

is satisfied for some $x_{0} \in D$, then

$$
\inf _{x \in D}\|f(x)-p x\|=0 .
$$

Proof. Consider a mapping $h: \operatorname{cl} D \times\langle 0,1\rangle \rightarrow X$ given by the formula

$$
h(x, t)=x-p^{-1} t\left(f(x)-p x_{0}\right) .
$$

Clearly, $\langle 0,1\rangle \ni t \rightarrow h(\cdot, t)$ is a continuous map into the space of $F$-mappings. Suppose that

$$
\inf _{x \in D}\|f(x)-p x\|>0
$$

If

$$
\inf _{t \in\langle 0,1\rangle} \inf _{x \in \mathrm{fr} D}\left\|h(x, t)-x_{0}\right\|>0
$$

then by Theorem 4(ii), $\operatorname{Deg}\left(h(\cdot, t), D x_{0}\right)$ does not depend on $t \in\langle 0,1\rangle$. Moreover, $\operatorname{Deg}\left(h(\cdot, 0) \cdot D \cdot x_{0}\right)=\mathbf{1} \in \mathscr{G}$ where 1 is represented by the constant sequence $1 \in Z$; therefore, by Theorem 5 (ii), there is $\left\{x_{n}\right\} \supset D$ such that

$$
x_{n}-p^{-1}\left(f(x)-p x_{0}\right) \rightarrow x_{0} .
$$

But this contradicts (17). Thus

$$
\inf _{t . x}\left\|h(x, t)-x_{0}\right\|=0 .
$$

By the compactness of $\langle 0,1\rangle$, there exists $t_{0} \in\langle 0,1\rangle$ such that

$$
\inf _{x \in \mathrm{fr} D}\left\|h\left(x, t_{0}\right)-x_{0}\right\|=0 .
$$

Due to (16), $t_{0}=1$ and this also contradicts (17). Hence (17) is false. Q.E.D.

Corollary 9. (i) If $I-p^{-1} f$ is a closed mapping, then the equation $f(x)=p x$ has a solution.

(ii) Having $p=1$, we obtain the existence of $\varepsilon$-fixed points of $f$.

We give three sufficient conditions for (16) to be satisfied. 
Theorem 10. Each of the following conditions:

(i) $D$ is convex and $p^{-1} f: \operatorname{cl} D \rightarrow \operatorname{cl} D$,

(ii) $\left\|p^{-1} f(x)-x_{0}\right\| \leq\left\|x-x_{0}\right\|$ for $x \in$ fr $D$,

(iii) $\left\|p^{-1} f(x)-x_{0}\right\|^{r} \leq\left\|p^{-1} f(x)-x\right\|^{r}+\left\|x-x_{0}\right\|^{r}$,

for a certain $r>1$ and each $x \in$ fr $D$, implies condition (16).

Proof. (i) Let $\varphi$ be the Minkowski gauge of the set $D-x_{0}$. Then, for any $t>0$ and $x \in$ fr $D$,

$$
\varphi\left(p^{-1} f(x)-x_{0}\right)-t \leq \varphi\left(p^{-1} f(x)-x_{0}-t\left(x-x_{0}\right)\right) .
$$

If we suppose that (16) does not hold, then there is $t>1$ with

$$
\inf _{x \in \operatorname{fr} D} \varphi\left(p^{-1} f(x)-x_{0}-t\left(x-x_{0}\right)\right)=0 .
$$

Therefore $\inf _{x \in \text { fr } D} \varphi\left(p^{-1} f(x)-x_{0}\right)>1$, which contradicts the condition that $p^{-1} f(\operatorname{cl} D) \subset \operatorname{cl} D$.

(ii) Suppose that there exists $t>1$ such that

$$
\inf _{x \in \operatorname{fr} D}\left\|p^{-1} f(x)-x_{0}-t\left(x-x_{0}\right)\right\|=0 .
$$

But this last norm is not less than

$$
\begin{aligned}
\left\|p^{-1} f(x)-x_{0}\right\|-t\left\|x-x_{0}\right\| & \geq(t-1)\left\|x-x_{0}\right\| \\
& \geq(t-1) d\left(x_{0}, \text { fr } D\right)>0 .
\end{aligned}
$$

(iii) Suppose that (16) does not hold. Then there are $t>1$ and a sequence $\left(x_{n}\right), x_{n} \in$ fr $D$, such that

$$
a_{n}-t c_{n} \rightarrow 0 \text { and } t b_{n}-(t-1) a_{n} \rightarrow 0
$$

where

$$
a_{n}=\left\|p^{-1} f\left(x_{n}\right)-x_{0}\right\|, \quad b_{n}=\left\|p^{-1} f\left(x_{n}\right)-x_{n}\right\|, \quad c_{n}=\left\|x_{n}-x_{0}\right\| .
$$

Using (iii), we get

$$
a_{n}^{r}\left[\left(t^{r}-1\right)-(t-1)^{r}\right] \leq\left(t b_{n}\right)^{r}-\left[(t-1) a_{n}\right]^{r}+\left(t c_{n}\right)^{r}-a_{n}^{r}
$$

and the right-hand side tends to 0 as $n \rightarrow \infty$. But $\left(t^{r}-1\right)-(t-1)^{r} \geq m>0$, so $a_{n} \rightarrow 0$, and this implies $c_{n} \rightarrow 0$, which is impossible. Q.E.D.

Theorems 8 and 10 and Corollary 9 constitute generalizations of the wellknown theorem of Schauder, Rothe, and Altman (see [5]).

Now we show a Borsuk-type theorem. It is based on the results of [6] and Jaworowski [8].

Theorem 11. Let $D$ be an open and bounded neighborhood of 0 in a Banach space $X$ with filtration $\left(X_{n}\right)$. Let $\gamma: \operatorname{fr} D \rightarrow \operatorname{fr} D$ be a continuous involution such that $\gamma\left(\operatorname{fr} D \cap X_{n}\right) \subset X_{n}$ for $n \geq n_{0} \quad\left(n_{0} \in N\right)$ and let $f$ be an F-mapping 
defined on $\mathrm{cl} D$, taking values in a Banach space $Y$ with filtration $\left(Y_{n}\right)$, such that

$$
0<\inf _{x \in \text { fr } D}\|f(x)\| \leq \sup _{x \in \text { fr } D}\|f(x)\|<\infty .
$$

If $f$ is an F-mapping with respect to $f \circ \gamma$ (i.e., $f-t(f \circ \gamma)$ is an F-map for $t \in\langle 0,1\rangle)$ as well, and

$$
\inf _{x \in \operatorname{fr} D, t \in\langle 0,1\rangle}\|f(x)-t f \circ \gamma(x)\|>0,
$$

then $\operatorname{Deg}(f, D, 0)=\left(\left(s_{n}\right)_{n=1}^{\infty}\right)$ where $s_{n} \equiv 1(\bmod 2)$ for $n \in N$.

Proof (cf. [8]). Let us assume that, for any $x \in \operatorname{fr} D,-f(x)=f \circ \gamma(x)$, and let $\left(f_{n}: \operatorname{cl} D \cap Y_{n} \rightarrow Y_{n}\right)$ be a sequence associated with the map $f$. As in the definition of the degree Deg, we prove that there exists an $n_{1} \geq n_{0}$ such that $0 \notin f_{n}\left(\right.$ fr $\left.D \cap X_{n}\right)$ for $n \geq n_{1}$. Let us define a sequence $\left(g_{n}:\right.$ fr $\left.D \cap X_{n} \rightarrow Y_{n}\right)$ by the formula

$$
g_{n}(x)=\frac{1}{2}\left(f_{n}(x)-f_{n} \circ \gamma(x)\right)
$$

for $x \in \operatorname{fr} D \cap X_{n}$. Let us note that

$$
\begin{aligned}
\sup _{x \in \mathrm{fr} D} & \left(\left\|g_{n}(x)-f(x)\right\|-d\left(f(x), Y_{n}\right)\right) \\
\leq & \frac{1}{2}\left[\sup \left(\left\|f_{n}(x)-f(x)\right\|-d\left(f(x), Y_{n}\right)\right)\right. \\
& \left.\quad+\sup \left(\left\|f_{n} \circ \gamma(x)-f \circ \gamma(x)\right\|-d\left(f \circ \gamma(x), Y_{n}\right)\right)\right] .
\end{aligned}
$$

This proves that the sequence $\left(g_{n}\right)$ is associated with $f$ on $\operatorname{fr} D$, too. Hence

$$
\operatorname{Deg}(f, D, 0)=\nu\left(\left(\operatorname{deg}\left(\tilde{g}_{n}\right), D \cap X_{n}, 0\right)_{n=1}^{\infty}\right)
$$

where $\tilde{g}_{n}$ is an arbitrary extension of $g_{n}$ onto $\operatorname{cl} D \cap X_{n}$. For each $n \geq n_{1}$, $\tilde{g}_{n}$ satisfies the assumptions of the Fet-Jaworowski theorem (see [6, 8]); thus $\operatorname{deg}\left(\tilde{g}_{n}, D \cap X_{n}, 0\right) \equiv 1(\bmod 2)$.

Let us come back to the general case. Consider a mapping $h:$ fr $D \times\langle 0,1\rangle \rightarrow$ $Y$ given by the formula

$$
h(x, t)=f(x)-t f \circ \gamma(x)
$$

for $x \in \operatorname{fr} D, t \in\langle 0,1\rangle$. Obviously, in view of the hypotheses of our theorem, a map $\langle 0,1\rangle \ni t \mapsto h(\cdot, t)$ satisfies the assumptions of Theorem 7(ii). Hence

$$
\operatorname{Deg}(f, D, 0)=\operatorname{Deg}(f-f \circ \gamma, D, 0)=\nu\left(\left(s_{n}\right)_{n=1}^{\infty}\right)
$$

where $s_{n} \equiv 1(\bmod 2)$ by the first part of the proof. Q.E.D.

As has been seen above, the preceding theorem possesses its equivalent for the class of $P F$-mappings. Moreover, let us note that the hypotheses of Theorem 11 can be immensely simplified (see [10]) in the case of $A$-mappings, for a linear combination of $A$-mappings is again an $A$-map. 


\section{REFERENCES}

1. L. E. J. Brouwer, Über Abbildung von Mannigfaltigkeiten, Math. Ann. 71 (1912), 97-115.

2. F. E. Browder, The degree of mapping, and its generalizations, Contemp. Math. 21 (1983), $15-40$.

3. F. E. Browder and W. V. Petryshyn, The topological degree and Galerkin approximation for noncompact operators in Banach spaces, Bull. Amer. Math. Soc. 74 (1968), 641-646.

4. __ Approximation methods and the generalized topological degree for nonlinear mappings in Banach spaces, J. Funct. Anal. 3 (1969), 217-245.

5. J. Dugundji and A. Granas, Fixed point theory, PWN, Warszawa, 1982.

6. A. I. Fet, Generalization of a theorem of Lusternik-Schnirelman of coverings of spheres and some theorems connected with it, Dokl. Akad. Nauk SSSR 95 (1954), 1149-1151.

7. P. M. Fitzpatrick, A generalized degree for uniform limits of A-proper mappings, J. Math. Anal. Appl. 35 (1971), 536-552.

8. J. W. Jaworowski, Involutions of compact spaces and a generalization of Borsuk's theorem on antipodes, Bull. Acad. Polon. Sci. 3 (1955), 289-292.

9. W. Kryszewski, Some remarks on the nonlinear eigenvalue problems of Birkhoff-Kellogg type, Bull. Acad. Polon. Sci. 32 (1984), 455-462.

10. __ An approximation method in the theory of nonlinear noncompact operators, C.R. Semi. Math. Sup. Col. A. Granas, Les Presses de l'Université de Montréal, 1986.

11. W. Kryszewski and B. Przeradzki, The topological degree and fixed points of DC-mappings, Fund. Math. 126 (1984).

12. A A new approximation method in the theory of nonlinear noncompact operators, Proc. Conf. Classical Analysis, Kazimierz Dolny, 1983.

13. J. Leray and J. Schauder, Topologie et équations fonctionelles, Ann. Sci. École Norm. Sup. 51 (1934), 45-78.

14. N. G. Lloyd, Degree theory, Cambridge Univ. Press, Cambridge, 1978.

15. T. Nirenberg, Topics in nonlinear functional analysis, New York Univ. Press, New York, 1974.

16. B. Nowak, DJ-odwzorowania i ich homotopie, Acta Univ. Lodziensis, Lódź, 1981.

17. R. D. Nussbaum, Degree theory for local condensing maps, J. Math. Anal. Appl. 37 (1972), 741-766.

18. W. V. Petryshyn, On the approximation solvability of equations involving A-proper and pseudo A-proper mappings, Bull. Amer. Math. Soc. 81 (1975), 223-312.

19. $\ldots$, On the approximation-solvability of nonlinear equations, Math. Ann. 177 (1968), 156164.

20. B. Przeradzki, On the homotopical classification of DJ-mappings of infinitely-dimensional spheres, Fund. Math. 120 (1984), 41-49.

21. B. N. Sadowski, Ultimately compact and condensing operators (Russian), Uspehi Mat. Nauk 27 (1971), 81-146.

22. I. V. Skripnik, Nonlinear elliptic equations of higher order, "Naukova Dumka", Kiev, 1973.

23. S. Werenski, On the fixed point index of noncompact mappings, Studia Math. 78 (1984), 155160.

Institute of Mathematics, University of Lodz, 90-238 Lodz, Poland 